\title{
Stability and Hopf Bifurcation Analysis in a Delayed Myc/E2F/miR-17-92 Network Involving Interlinked Positive and Negative Feedback Loops
}

\author{
Guiyuan Wang (D) and Zhuoqin Yang (D) \\ School of Mathematics and Systems Science and LMIB, Beihang University, Beijing 100191, China \\ Correspondence should be addressed to Zhuoqin Yang; yangzhuoqin@buaa.edu.cn
}

Received 24 July 2018; Accepted 17 October 2018; Published 11 November 2018

Academic Editor: Youssef N. Raffoul

Copyright (c) 2018 Guiyuan Wang and Zhuoqin Yang. This is an open access article distributed under the Creative Commons Attribution License, which permits unrestricted use, distribution, and reproduction in any medium, provided the original work is properly cited.

\begin{abstract}
MiR-17-92 plays an important role in regulating the levels of the Myc/E2F protein. In this paper, we consider a coupling network between Myc/E2F/miR-17-92 delayed negative feedback loop and Myc/E2F positive feedback loop described by a two-dimensional delay differential equation. Based on linear stability analysis and bifurcation theory, sufficient conditions for stability of equilibria and oscillatory behaviors via Hopf bifurcation are derived when choosing time delay as well as negative feedback strength associated with oscillations as bifurcation parameters, respectively. Furthermore, direction and stability of Hopf bifurcation of time delay are studied by using the normal form method and center manifold theorem. Finally, several numerical simulations are performed to verify the results we obtained.
\end{abstract}

\section{Introduction}

Due to the development of large-scale experimental and computational techniques, a posttranscriptional regulation by small noncoding microRNAs (miRNAs) has been discovered in many cellular processes, including cell growth, development, differentiation, and apoptosis [1-4]. The miR-17-92 cluster as a polycistronic gene located in human chromosome 13 ORF 25 (c13orf25) is composed of 7 mature miRNAs [1]. MiRNAs play critical roles in biological processes, as posttranscriptional regulators of gene expression [2]. MicroRNAbased regulation has been simulated by specially designed mathematical models [5-7]. The transcription factors E2F and Myc act as tumor suppressors or oncogenes and participate in the control of cell proliferation and apoptosis [1]. Aguda et al. proposed a simple model involving miR-17-92, E2F, and Myc, which is composed of a positive feedback (E2F/Myc) and a negative feedback (Myc/E2F/miR-17-92) [1]. It presents a bistable switch behavior and a one-way switch in the network, which corresponds to the bistability and monostability, respectively $[1,2]$. Subsequently, Li et al. illustrated an abstract model of the network presented by
Aguda et al. and focused on the physiological significance of miRNAs [2]. It was found that the existence of miRNAs improves the ability of the bistable switches in the network [2]. Zhang et al. further analyzed this abstract model and suggested that the interlinked positive and negative feedback loops buffer noise effects rather than only amplifying or suppressing the noise [3].

Dynamical analysis of the system with time delay is an essential topic in many fields, especially for the models of gene expression (see [8-11]). Time delay is inevitable in Myc/E2F/miR-17-92 network since feedback loops involve many intermediate processes such as transcription, translation, and posttranslational modifications [12-14]. Time delay influences the time-dependent dynamics even for the simplest circuits with one and two gene elements, which can give rise to rich dynamical behaviors such as periodic and chaotic dynamics [15].

In this work, we consider the effect of time delay in the delayed Myc/E2F /miR-17-92 network. As negative feedback is often used in biochemistry to generate oscillations to achieve homeostasis, the steady state may lose stability and be replaced by oscillations [16]. So an inhibition efficiency 


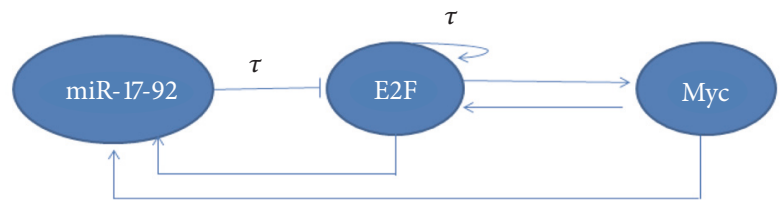

(a)

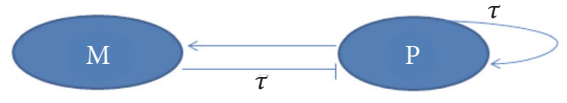

(b)

FIGURE 1: An illustration of the network involving miR-17-92, E2F and Myc (a) and its abstract model (b), where P and M represent the protein module (E2Fs and Myc) and miR-17-92 cluster, respectively.

parameter $\Gamma_{2}$ in the network is incorporated into ordinary differential equations to give rise to periodic solutions at certain critical values.

The organization of the paper is as follows: we describe the $\mathrm{Myc} / \mathrm{E} 2 \mathrm{~F} / \mathrm{miR}-17-92$ network with time delay in Section 2. In Section 3.1, we discuss local stability of the solution and Hopf bifurcation of the network with respect to negative feedback strength $\Gamma_{2}$. Under different values of $\Gamma_{2}$, local stability and Hopf bifurcation of time delay are studied in Section 3.2. In Section 3.3, a formula is given to determine the direction and stability of the Hopf bifurcation of the time delay. In Section 4, four examples on influence of time delay on different states of negative feedback strength $\Gamma_{2}$ are given to support our results. Finally, the conclusions are drawn in Section 5.

\section{Model Description}

An illustration of network involving miR-17-92, E2F, and Myc and abstract structure of this network are depicted in Figure 1, where $\mathrm{P}$ is the protein module and $\mathrm{M}$ denotes the miRNA cluster module. The positive feedback in module $\mathrm{P}$ (E2Fs and $\mathrm{Myc}$ ) as an autocatalytic process can lead to the transcription of $\mathrm{M}$ but be inhibited by $\mathrm{M}$.

Aguda B D et al. presented a model of interaction between miR-17-92, E2F and Myc, which is described by the following differential equations [1]:

$$
\begin{aligned}
& \frac{d[P]}{d t}=\alpha_{p}+\frac{k_{p}[P]^{2}}{\Gamma_{1}+[P]^{2}+\Gamma_{2}[M]}-\beta_{p}[P], \\
& \frac{d[M]}{d t}=\alpha_{M}+k_{M}[P]-\beta_{M}[M],
\end{aligned}
$$

where $[\mathrm{P}]$ and $[\mathrm{M}]$ represent the concentrations of $\mathrm{P}$ and $\mathrm{M}$, respectively. $\alpha_{M}$ denotes the P-independent constitutive transcription of $\mathrm{M}$, and $\alpha_{p}$ describes the constitutive protein expression. Parameters $\beta_{p}$ and $\beta_{M}$ are the degradation rates of $\mathrm{P}$ and $\mathrm{M}$, respectively. $\Gamma_{1}$ is the coefficient of protein expression and $\Gamma_{2}$ is the inhibition efficiency parameter. $k_{M}$ stands for the rate constant, and $k_{P}$ represents the constant of protein expression.

For this model, we consider the inhibition efficiency parameter $\Gamma_{2}$ to explore stability of equilibria and oscillatory behaviors via Hopf bifurcation through its critical value as negative feedback usually leads to oscillation. Moreover, we investigate the effect of the time delay in the network when the concentrations of $\mathrm{P}$ and $\mathrm{M}$ are described by the following delayed differential equations (DDEs):

$$
\begin{aligned}
\frac{d[P]}{d t}= & \alpha_{p}+\frac{k_{p}[P(t-\tau)]^{2}}{\Gamma_{1}+[P(t-\tau)]^{2}+\Gamma_{2}[M(t-\tau)]} \\
& -\beta_{p}[P], \\
\frac{d[M]}{d t}= & \alpha_{M}+k_{M}[P]-\beta_{M}[M] .
\end{aligned}
$$

For the convenience of analysis, we define

$$
\begin{aligned}
P & =[P] \\
\text { and } M & =[M] .
\end{aligned}
$$

With these substitutions, system (2) can be rewritten as

$$
\begin{aligned}
& \frac{d P}{d t}=\alpha_{p}+\frac{k_{p} P^{2}(t-\tau)}{\Gamma_{1}+P^{2}(t-\tau)+\Gamma_{2} M(t-\tau)}-\beta_{p} P, \\
& \frac{d M}{d t}=\alpha_{M}+k_{M} P-\beta_{M} M .
\end{aligned}
$$

The initial values for system (4) take the form of

$$
\begin{aligned}
& P(\theta)=\varphi_{1}(\theta) \geq 0, \\
& M(\theta)=\varphi_{2}(\theta) \geq 0, \\
& \quad \theta \in[-\tau, 0), \\
& \varphi_{1}(0)=P(0)>0, \\
& \varphi_{2}(0)=M(0)>0,
\end{aligned}
$$

where $\left(\varphi_{1}, \varphi_{2}\right) \in C\left([-\tau, 0],[0,+\infty)^{2}\right)$, and all the parameters are positive.

\section{Main Results}

System (4) exhibits dynamics behaviors of the steady state and periodic phenomenon in the different parameter regimes. Here, we focus especially on theoretical analysis on the stability and existence of Hopf bifurcation of system (4).

3.1. Local Stability and Hopf Bifurcation of the Negative Feedback Strength $\Gamma_{2}$. Firstly, at the time delay $\tau=0$, we consider 
Hopf bifurcation of the negative feedback strength $\Gamma_{2}$ as the measure of the miRNA inhibition through the critical values and local stability of equilibrium before the bifurcation in system (4).

Let $E_{*}\left(P_{*}, M_{*}\right)$ be the equilibrium of system (4), and then

$$
\begin{aligned}
\alpha_{p}+\frac{k_{p} P_{*}^{2}}{\Gamma_{1}+P_{*}^{2}+\Gamma_{2} M_{*}}-\beta_{p} P_{*} & =0, \\
\alpha_{M}+k_{M} P_{*}-\beta_{M} M_{*} & =0 .
\end{aligned}
$$

Eliminating $M_{*}$ from above equations, we get the following equation on $P_{*}$ :

$$
\begin{gathered}
\beta_{M} \beta_{p} P_{*}^{3}+\left(k_{M} \Gamma_{2} \beta_{p}-\beta_{M}\left(\alpha_{p}+k_{p}\right)\right) P_{*}^{2} \\
+\left(\beta_{M} \Gamma_{1} \beta_{p}+\Gamma_{2}\left(\alpha_{M} \beta_{p}-k_{M} \alpha_{p}\right)\right) P_{*} \\
-\alpha_{p}\left(\beta_{M} \Gamma_{1}+\Gamma_{2} \alpha_{M}\right)=0 .
\end{gathered}
$$

Obviously, the equation has at most three positive real roots. Setting $E_{*}\left(P_{*}, M_{*}\right)$ is one of the three roots.

Secondly, we translate the equilibrium $E_{*}$ to the origin. By the linear transform

$$
\begin{aligned}
& x(t)=P(t)-P_{*} \\
& y(t)=M(t)-M_{*}
\end{aligned}
$$

system (4) becomes

$$
\begin{aligned}
\dot{x}(t)= & m x(t-\tau)-n y(t-\tau)-\beta_{p} x \\
& +\sum_{i+j \geq 2} \frac{1}{i ! j !} f_{i j}^{(1)} x^{i}(t-\tau) y^{j}(t-\tau), \\
\dot{y}(t)= & k_{M} x(t)-\beta_{M} y(t),
\end{aligned}
$$

where

$$
\begin{aligned}
m & =\frac{2 k_{p} P_{*}\left(\Gamma_{1}+\Gamma_{2} M_{*}\right)}{\left(\Gamma_{1}+P_{*}{ }^{2}+\Gamma_{2} M_{*}\right)^{2}}, \\
n & =\frac{k_{p} P_{*}{ }^{2} \Gamma_{2}}{\left(\Gamma_{1}+P_{*}{ }^{2}+\Gamma_{2} M_{*}\right)^{2}}, \\
f^{(1)} & =\alpha_{p}+\frac{k_{p} P^{2}(t-\tau)}{\Gamma_{1}+P^{2}(t-\tau)+\Gamma_{2} M(t-\tau)}-\beta_{p} P, \\
f^{(2)} & =\alpha_{M}+k_{M} P-\beta_{M} M, \\
f_{i j}^{(1)} & =\left.\frac{\partial^{i+j} f^{(1)}}{\partial x^{i} \partial y^{j}}\right|_{\left(P_{*}, M_{*}\right)}, \quad i, j \geq 0 .
\end{aligned}
$$

From (9), it is easy to get the following characteristic equation. That is,

$$
\left|\begin{array}{cc}
\lambda-m+\beta_{p} & n \\
-k_{M} & \lambda+\beta_{M}
\end{array}\right|=0
$$

thus, the two degree polynomial equation is obtained

$$
\lambda^{2}+\left(\beta_{M}+\beta_{p}-m\right) \lambda+\beta_{M}\left(\beta_{p}-m\right)+n k_{M}=0 .
$$

If

$$
\begin{array}{r}
\beta_{M}+\beta_{p}-m>0, \\
\beta_{M}\left(\beta_{p}-m\right)+n k_{M}>0
\end{array}
$$

then all the roots of (12) have negative real parts. So the equilibrium $E_{*}$ of system (4) is locally stable.

On the other hand, we assume

$$
\beta_{M}+\beta_{p}-m=0
$$

and $\lambda_{1,2}(\mu)=\alpha(\mu) \pm i \omega(\mu)$ are the roots of $(12)$.

Viewing the negative feedback strength $\Gamma_{2}$ as a bifurcation parameter, the sufficient conditions for the occurrence of the Hopf bifurcation of system (4) are obtained in the following results.

Theorem 1. If (H2) and (H3) hold, there exist $\Gamma_{2}=\Gamma_{2}^{0}$, such that

$$
\begin{aligned}
\alpha\left(\Gamma_{2}^{0}\right) & =0, \\
\omega\left(\Gamma_{2}^{0}\right) & >0, \\
\frac{d \alpha\left(\Gamma_{2}^{0}\right)}{d \Gamma_{2}} & \neq 0,
\end{aligned}
$$

then system (4) can exhibit Hopf bifurcation.

Time delay is inevitable and plays an important role in negative feedback loop of the Myc/E2F/miR-17-92 network due to the transcription and translation. So time delay on this network is considered in the next sections.

3.2. Local Stability and Hopf Bifurcation of Time Delay. In this section, we take the time delay $\tau$ as a bifurcating parameter to investigate the stability and existence of Hopf bifurcation of time delay in system (4). For convenience, let $k_{1}=k_{p}, k_{2}=$ $\beta_{p}, k_{3}=k_{M}, k_{4}=\beta_{M}$, then system (9) becomes

$$
\begin{aligned}
\dot{x}(t)= & m x(t-\tau)-n y(t-\tau)-k_{2} x \\
& +\sum_{i+j \geq 2} \frac{1}{i ! j !} f_{i j}^{(1)} x^{i}(t-\tau) y^{j}(t-\tau), \\
\dot{y}(t)= & k_{3} x-k_{4} y,
\end{aligned}
$$

where

$$
\begin{aligned}
& m=k_{1} n_{1}, \\
& n=k_{1} n_{2}, \\
& n_{1}=\frac{2 x_{*}\left(\Gamma_{1}+\Gamma_{2} y_{*}\right)}{\left(\Gamma_{1}+x_{*}^{2}+\Gamma_{2} y_{*}\right)^{2}}, \\
& n_{2}=\frac{\Gamma_{2} x_{*}{ }^{2}}{\left(\Gamma_{1}+x_{*}{ }^{2}+\Gamma_{2} y_{*}\right)^{2}} .
\end{aligned}
$$


Then we obtain the characteristic equation of system (14).

That is,

$$
\begin{gathered}
\lambda^{2}+\left(k_{2}+k_{4}\right) \lambda-k_{1} n_{1} e^{-\lambda \tau} \lambda+k_{2} k_{4} \\
+\left(k_{1} k_{3} n_{2}-k_{1} k_{4} n_{1}\right) e^{-\lambda \tau}=0
\end{gathered}
$$

When $\tau=0$, it becomes

$$
\begin{aligned}
\lambda^{2} & +\left(k_{2}+k_{4}-k_{1} n_{1}\right) \lambda+k_{2} k_{4}+k_{1} k_{3} n_{2}-k_{1} k_{4} n_{1} \\
& =0 .
\end{aligned}
$$

$i \omega(\omega>0)$ is the root of (16) if and only if $i \omega$ satisfies

$$
\begin{aligned}
-\omega^{2} & +\left(k_{2}+k_{4}\right)(i \omega) \\
& -k_{1} n_{1}(\cos (\omega \tau)-i \sin (\omega \tau))(i \omega)+k_{2} k_{4} \\
& +\left(k_{1} k_{3} n_{2}-k_{1} k_{4} n_{1}\right)(\cos (\omega \tau)-i \sin (\omega \tau))=0 .
\end{aligned}
$$

Separating the real and imaginary parts, we get

$$
\begin{aligned}
\left(k_{2}+k_{4}\right) \omega= & k_{1} n_{1} \omega \cos (\omega \tau) \\
& +\left(k_{1} k_{3} n_{2}-k_{1} k_{4} n_{1}\right) \sin (\omega \tau), \\
k_{2} k_{4}-\omega^{2}= & k_{1} n_{1} \omega \sin (\omega \tau) \\
& -\left(k_{1} k_{3} n_{2}-k_{1} k_{4} n_{1}\right) \cos (\omega \tau) .
\end{aligned}
$$

which is equivalent to

$$
\begin{aligned}
\omega^{4} & -\left(k_{1}^{2} n_{1}^{2}-k_{2}^{2}-k_{4}^{2}\right) \omega^{2}+k_{2}^{2} k_{4}^{2}-k_{1}^{2}\left(k_{3} n_{2}-k_{4} n_{1}\right)^{2} \\
& =0 .
\end{aligned}
$$

Let $z=\omega^{2}$, then it is transformed to be

$$
z^{2}-p z+q=0
$$

where

$$
\begin{aligned}
& p=k_{1}^{2} n_{1}^{2}-k_{2}^{2}-k_{4}^{2}, \\
& q=k_{2}^{2} k_{4}^{2}-k_{1}^{2}\left(k_{3} n_{2}-k_{4} n_{1}\right)^{2} .
\end{aligned}
$$

Assume (H4) equation (21) has at least one positive real root.

From (21), we can obtain

$$
z_{1,2}=\frac{1}{2} p \pm \frac{1}{2} \sqrt{p^{2}-4 q}
$$

Thus, if

$$
\begin{array}{r}
p<0, \\
q>0 \\
\text { or } p^{2}-4 q<0
\end{array}
$$

then none of $z_{1}, z_{2}$ is positive. So (21) has no positive roots. It means that there are no imaginary roots in characteristic equation (16).
From those discussed above, we obtain

$$
\begin{aligned}
& \cos (\omega \tau)=\frac{k_{2} k_{4}^{2} n_{1}+\left(k_{3} n_{2}+k_{2} n_{1}\right) \omega^{2}-k_{2} k_{3} k_{4} n_{2}}{k_{1}\left(\left(k_{3} n_{2}-k_{4} n_{1}\right)^{2}+n_{1}^{2} \omega^{2}\right)}, \\
& \sin (\omega \tau)=\frac{\omega\left(k_{2} k_{3} n_{2}+k_{3} n_{2} k_{4}-n_{1} \omega^{2}-n_{1} k_{4}^{2}\right)}{k_{1}\left(\left(k_{3} n_{2}-k_{4} n_{1}\right)^{2}+n_{1}^{2} \omega^{2}\right)} .
\end{aligned}
$$

Without loss of generality, (21) is assumed to have two positive roots, defined by $z_{1}, z_{2}$, respectively. Thus, (20) has two positive roots $\omega_{1}=\sqrt{z_{1}}, \omega_{2}=\sqrt{z_{2}}$.

So we have

$$
\cos \left(\omega_{k} \tau_{k}\right)=\frac{k_{2} k_{4}^{2} n_{1}+\left(k_{3} n_{2}+k_{2} n_{1}\right) \omega_{k}^{2}-k_{2} k_{3} k_{4} n_{2}}{k_{1}\left(\left(k_{3} n_{2}-k_{4} n_{1}\right)^{2}+n_{1}^{2} \omega_{k}^{2}\right)} .
$$

It follows that

$$
\tau_{k}^{j}=\frac{1}{\omega_{k}}
$$

$$
\begin{aligned}
& \cdot \arccos \left(\frac{k_{2} k_{4}^{2} n_{1}+\left(k_{3} n_{2}+k_{2} n_{1}\right) \omega_{k}^{2}-k_{2} k_{3} k_{4} n_{2}}{k_{1}\left(\left(k_{3} n_{2}-k_{4} n_{1}\right)^{2}+n_{1}^{2} \omega_{k}^{2}\right)}\right) \\
& +\frac{2 j \pi}{\omega_{k}},
\end{aligned}
$$

where $k=1,2, j=0,1,2, \ldots$

Define

$$
\tau_{0}=\min \left\{\tau_{1}^{0}, \tau_{2}^{0}\right\}
$$

Taking the derivative of $\lambda$ with respect to $\tau$, we can get

$$
\left[\frac{d \lambda}{d \tau}\right]^{-1}=\frac{2 \lambda+\left(k_{2}+k_{4}\right)-k_{1} n_{1} e^{-\lambda \tau}}{k_{1} \lambda e^{-\lambda \tau}\left(k_{3} n_{2}-k_{4} n_{1}-n_{1} \lambda\right)}-\frac{\tau}{\lambda} .
$$

Then,

$$
\operatorname{Re}\left[\frac{d \lambda}{d \tau}\right]_{\tau=\tau_{0}}^{-1}=\frac{c+d}{a^{2}+b^{2}}
$$

where

$$
\begin{aligned}
& a=k_{1} k_{3} n_{2}-k_{1} k_{4} n_{1}, \\
& \mathrm{~b}=k_{1} n_{1} \omega_{0}, \\
& c=2\left(\cos \left(\omega_{0} \tau_{0}\right) a-\sin \left(\omega_{0} \tau_{0}\right) b\right)-k_{1}{ }^{2} n_{1}{ }^{2}, \\
& d=\left(k_{2}+k_{4}\right)\left[k_{1} n_{1} \cos \left(\omega_{0} \tau_{0}\right)+\frac{1}{\omega_{0}} a \sin \left(\omega_{0} \tau_{0}\right)\right] .
\end{aligned}
$$

If the condition

$$
c+d \neq 0
$$

holds, then

$$
\operatorname{Re}\left[\frac{d \lambda}{d \tau}\right]_{\tau=\tau_{0}}^{-1} \neq 0 .
$$

That is, the transversality condition is satisfied.

Based on the analysis above, the following results are obtained. 
Theorem 2. Let $\tau=\tau_{k}^{j}$ be defined by (26); one has

(i) if (H1), (H2), and (H5) hold, then the equilibrium $E_{*}$ of system (4) is asymptotically stable for all $\tau \geq 0$;

(ii) if (H1), (H2), and (H4) hold, then the equilibrium $E_{*}$ is locally asymptotically stable for $0 \leq \tau<\tau_{0}$ and unstable for $\tau>\tau_{0}$; moreover, if $c+d \neq 0$, then system (4) undergoes a Hopf bifurcation at the equilibrium $E_{*}$ when $\tau=\tau_{k}^{j}(k=1,2, j=$ $0,1,2, \ldots)$.

3.3. Direction and Stability of the Hopf Bifurcation at Time Delay $\tau=\tau_{0}$. In this section, we further consider direction and stability of Hopf bifurcation assuming at $\tau=\tau_{0}$ by using the methods in Hassard et al. [17]. For convenience, let $\tau=$ $\tau_{0}+\mu, \mu \in R, s=t / \tau$ and denote $s=t$, then system (14) becomes

$$
\begin{aligned}
& \dot{x}(t)=\left(\tau_{0}+\mu\right)\left\{m x(t-1)-n y(t-1)-k_{2} x\right. \\
& \left.\quad+\sum_{i+j \geq 2} \frac{1}{i ! j !} f_{i j}^{(1)} x^{i}(t-1) y^{j}(t-1)\right\}, \\
& \dot{y}(t)=\left(\tau_{0}+\mu\right)\left\{k_{3} x(t)-k_{4} y(t)\right\} .
\end{aligned}
$$

Clearly, $\mu=0$ is a Hopf bifurcation value of system (32). Its linear part is given by

$$
\begin{aligned}
& \dot{x}(t)=\left(\tau_{0}+\mu\right)\left\{m x(t-1)-n y(t-1)-k_{2} x(t)\right\}, \\
& \dot{y}(t)=\left(\tau_{0}+\mu\right)\left\{k_{3} x(t)-k_{4} y(t)\right\} .
\end{aligned}
$$

and nonlinear part is

$$
\left.\begin{array}{l}
f\left(\mu, u_{t}\right) \\
\quad=\left(\tau_{0}+\mu\right)\left[\sum_{i+j \geq 2} \frac{1}{i ! j !} f_{i j}^{(1)} x^{i}(t-1) y^{j}(t-1)\right] \\
0
\end{array}\right] .
$$

For convenience of studying the problems of Hopf bifurcation, we rewrite system (32) as

$$
\dot{u}(t)=L_{\mu} u_{t}+f\left(\mu, u_{t}\right)
$$

where $u(t)=(x(t), y(t))^{T}$ and $u_{t}(\theta)=u(t+\theta)$ for $\theta \in[-\tau, 0]$; $L_{\mu}: C \longrightarrow R, f: R \times C \longrightarrow R$ are given, respectively, by

$$
\begin{aligned}
L_{\mu}(\varphi) & =\left(\tau_{0}+\mu\right)\left[\begin{array}{cc}
-k_{2} & 0 \\
k_{3} & -k_{4}
\end{array}\right]\left[\begin{array}{l}
\varphi_{1}(0) \\
\varphi_{2}(0)
\end{array}\right]+\left(\tau_{0}+\mu\right)\left[\begin{array}{cc}
m & -n \\
0 & 0
\end{array}\right]\left[\begin{array}{c}
\varphi_{1}(-1) \\
\varphi_{2}(-1)
\end{array}\right], \\
f\left(\mu, u_{t}\right) & =\left(\tau_{0}+\mu\right)\left[\begin{array}{c}
\left.\sum_{i+j \geq 2} \frac{1}{i ! j !} f_{i j}^{(1)} x^{i}(t-1) y^{j}(t-1)\right] \\
0
\end{array}\right] \\
& =\left(\tau_{0}+\mu\right)\left[\begin{array}{c}
\left.\frac{1}{2} f_{20}^{(1)} \varphi_{1}^{2}(-1)+f_{11}^{(1)} \varphi_{1}(-1) \varphi_{2}(-1)+\frac{1}{2} f_{02}^{(1)} \varphi_{2}^{2}(-1)+\text { h.o.t. }\right] . \\
0
\end{array} .\right.
\end{aligned}
$$

Here, h.o.t. denotes higher order terms.

For convenience analysis, let

$$
\begin{aligned}
& L_{20}=\frac{1}{2} f_{20}^{(1)}, \\
& L_{11}=f_{11}^{(1)}, \\
& L_{02}=\frac{1}{2} f_{02}^{(1)},
\end{aligned}
$$

where $f_{20}^{(1)}=2 k_{1}\left(\Gamma_{1}+\Gamma_{2} y_{*}\right)\left(\Gamma_{1}-3 x_{*}^{2}+\Gamma_{2} y_{*}\right) /\left(\Gamma_{1}+x_{*}^{2}+\Gamma_{2} y_{*}\right)^{3}$; $f_{11}^{(1)}=-2 k_{1} x_{*} \Gamma_{2}\left(\Gamma_{1}-x_{*}^{2}+\Gamma_{2} y_{*}\right) /\left(\Gamma_{1}+x_{*}^{2}+\Gamma_{2} y_{*}\right)^{3}, f_{02}^{(1)}=$ $2 k_{1} x_{*}^{2} \Gamma_{2}^{2} /\left(\Gamma_{1}+x_{*}^{2}+\Gamma_{2} y_{*}\right)^{3}$.

Then,

$$
\begin{aligned}
& f\left(\mu, u_{t}\right)=\left(\tau_{0}+\mu\right) \\
& \quad \cdot\left[\begin{array}{c}
L_{20} \varphi_{1}^{2}(-1)+L_{11} \varphi_{1}(-1) \varphi_{2}(-1)+L_{02} \varphi_{2}^{2}(-1)+\text { h.o.t. } \\
0
\end{array}\right] .
\end{aligned}
$$

According to the Riesz representation theorem, there exists a $2 \times 2$ matrix function $\eta(\theta, \mu), \theta \in[-1,0]$, such that

$$
L_{\mu}(\varphi)=\int_{-1}^{0} d \eta(\theta, \mu) \varphi(\theta), \quad \varphi \in C^{1}\left([-1,0], R^{2}\right) .
$$

Here, we choose

$$
\begin{aligned}
\eta(\theta, \mu)= & \left(\tau_{0}+\mu\right)\left[\begin{array}{cc}
-k_{2} & 0 \\
k_{3} & -k_{4}
\end{array}\right] \delta(\theta) \\
& -\left(\tau_{0}+\mu\right)\left[\begin{array}{cc}
m & -n \\
0 & 0
\end{array}\right] \delta(\theta+1),
\end{aligned}
$$

where $\delta(\theta)$ is defined by

$$
\delta(\theta)= \begin{cases}0, & \theta \neq 0 \\ 1, & \theta=0\end{cases}
$$


For $\varphi \in C^{1}\left([-1,0], R^{2}\right)$, define

$$
A(\mu) \varphi= \begin{cases}\frac{d \varphi(\theta)}{d \theta}, & -1 \leq \theta<0, \\ \int_{-1}^{0} d \eta(\theta, \mu) \varphi(\theta), & \theta=0,\end{cases}
$$

and

$$
\begin{aligned}
& R(\mu) \varphi \\
& = \begin{cases}\left(\begin{array}{l}
0 \\
0
\end{array}\right), & -1 \leq \theta<0, \\
\left(\tau_{0}+\mu\right)\left(\begin{array}{cl}
\sum_{i+j \geq 2} \frac{1}{i ! j !} f_{i j}^{(1)} x^{i}(t-1) y^{j}(t-1) \\
0
\end{array}\right), & \theta=0 .\end{cases}
\end{aligned}
$$

For $\psi \in C^{1}\left([0,1],\left(R^{2}\right)^{*}\right)$, the adjoint operator $A^{*}$ of $A$ is defined by

$$
A^{*} \psi(s)= \begin{cases}-\frac{d \psi(s)}{d s}, & 0<s \leq 1, \\ \int_{-1}^{0} d \eta^{T}(s, \mu) \psi(-s), & s=0 .\end{cases}
$$

And define a bilinear inner product as follows:

$$
\begin{aligned}
\langle\psi(s), \varphi(\theta)\rangle= & \bar{\psi}(0) \varphi(0) \\
& -\int_{-1}^{0} \int_{\xi=0}^{\theta} \bar{\psi}(\xi-\theta) d \eta(\theta) \varphi(\xi) d \xi,
\end{aligned}
$$

where $\eta(\theta)=\eta(\theta, 0)$.

From the discussion in Section 3.2, we obtain that $\pm i \tau_{0} \omega_{0}$ are eigenvalues of $A(0)$. At the same time, they are also eigenvalues of $A^{*}$ since $A$ and $A^{*}$ are adjoint operator. In the following, we calculate the eigenvectors of $A(0)$ and $A^{*}$ corresponding to $i \tau_{0} \omega_{0}$ and $-i \tau_{0} \omega_{0}$, respectively. Suppose $q(\theta)=$ $(1, \beta)^{\mathrm{T}} e^{i \tau_{0} \omega_{0} \theta}, \theta \in[-1,0]$ is eigenvector of $A(0)$. Through the discussion above, we have

$$
A(0) q(0)=i \tau_{0} \omega_{0} q(0) .
$$

That is,

$$
\tau_{0}\left[\begin{array}{cc}
i \omega_{0}+k_{2}-m e^{-i \tau_{0} \omega_{0}} & n e^{-i \tau_{0} \omega_{0}} \\
-k_{3} & i \omega_{0}+k_{4}
\end{array}\right] q(0)=\left[\begin{array}{l}
0 \\
0
\end{array}\right] .
$$

We can obtain

$$
\begin{aligned}
\beta & =\frac{k_{3}}{i \omega_{0}+k_{4}} \\
\text { or } \beta & =\frac{m e^{-i \tau_{0} \omega_{0}}-i \omega_{0}-k_{2}}{n e^{-i \tau_{0} \omega_{0}}} .
\end{aligned}
$$

Thus, $q(0)=(1, \beta)^{\mathrm{T}}=\left(1, k_{3} /\left(i \omega_{0}+k_{4}\right)\right)^{\mathrm{T}}$ or $q(0)=(1$, $\left.\left(m e^{-i \tau_{0} \omega_{0}}-i \omega_{0}-k_{2}\right) / n e^{-i \tau_{0} \omega_{0}}\right)^{\mathrm{T}}$.

On the other hand, we assume $q^{*}(s)=D\left(1, \beta^{*}\right)^{\mathrm{T}} e^{i \tau_{0} \omega_{0} s}$, $s \in[0,1]$ is the eigenvector of $A^{*}$ and then obtain

$$
\tau_{0}\left[\begin{array}{cc}
-i \omega_{0}+k_{2}-m e^{-i \tau_{0} \omega_{0}} & -k_{3} \\
n e^{-i \tau_{0} \omega_{0}} & -i \omega_{0}+k_{4}
\end{array}\right] q^{*}(0)=\left[\begin{array}{l}
0 \\
0
\end{array}\right] .
$$

and

$$
\begin{gathered}
q^{*}(s)=D\left(1, \beta^{*}\right)^{\mathrm{T}}=D\left(1, \frac{n e^{-i \tau_{0} \omega_{0}}}{\left(i \omega_{0}-k_{4}\right)}\right) \\
\operatorname{or} q^{*}(s)=D\left(1, \frac{\left(k_{2}-i \omega_{0}-m e^{-i \tau_{0} \omega_{0}}\right)}{k_{3}}\right) .
\end{gathered}
$$

Then, normalize $q$ and $q^{*}$ by the following conditions:

$$
\begin{aligned}
& \left\langle q^{*}, q\right\rangle=1, \\
& \left\langle q^{*}, \bar{q}\right\rangle=0 .
\end{aligned}
$$

In order to get $\left\langle q^{*}(s), q(\theta)\right\rangle=1$, we need to determine the value of $D$.

$$
\begin{aligned}
& \left\langle q^{*}(s), q(\theta)\right\rangle=\bar{q}^{*}(0) \cdot q(0)-\int_{\theta=-1}^{0} \int_{\xi=0}^{\theta} \bar{q}^{* \mathrm{~T}}(\xi \\
& -\theta) d \eta(\theta) q(\xi) d \xi \\
& =\bar{D}\left(1, \bar{\beta}^{*}\right)(1, \beta)^{\mathrm{T}}-\int_{-1}^{0} \int_{\xi=0}^{\theta} \bar{D}\left(1, \bar{\beta}^{*}\right) \\
& \cdot e^{-i(\xi-\theta) \tau_{0} \omega_{0}} d \eta(\theta)(1, \beta)^{\mathrm{T}} e^{-i \xi \tau_{0} \omega_{0}} d \xi=\bar{D}\left\{1+\beta \bar{\beta}^{*}\right. \\
& \left.-\int_{-1}^{0}\left(1, \bar{\beta}^{*}\right) \theta e^{i \theta \tau_{0} \omega_{0}} d \eta(\theta)(1, \beta)^{\mathrm{T}}\right\}=\bar{D}\{1 \\
& \left.+\beta \bar{\beta}^{*}-\tau_{0}(n \beta-m) e^{-i \tau_{0} \omega_{0}}\right\}=1 .
\end{aligned}
$$

Thus, we choose $D$ as

$$
D=\frac{1}{\left(1+\bar{\beta} \beta^{*}-\tau_{0}(n \beta-m) e^{i \tau_{0} \omega_{0}}\right)}
$$

such that $\left\langle q^{*}, q\right\rangle=1,\left\langle q^{*}, \bar{q}\right\rangle=0$.

Next, we employ the algorithms in [17] and a computation process similar to [18-20] to calculate some important coefficients as follows:

$$
\begin{aligned}
g_{20} & =2 \tau_{0} \bar{D}\left\{L_{20}\left[q^{(1)}(-1)\right]^{2}+L_{02}\left[q^{(2)}(-1)\right]^{2}\right. \\
& \left.+L_{11} q^{(1)}(-1) q^{(2)}(-1)\right\} \\
g_{11} & =\tau_{0} \bar{D}\left\{2 L_{20} q^{(1)}(-1) \bar{q}^{(1)}(-1)+2 L_{02} q^{(2)}(-1)\right. \\
& \left.\cdot \bar{q}^{(2)}(-1)+L_{11} A_{11}\right\} \\
g_{02} & =2 \tau_{0} \bar{D}\left\{L_{20}\left[\bar{q}^{(1)}(-1)\right]^{2}+L_{02}\left[\bar{q}^{(2)}(-1)\right]^{2}\right. \\
& \left.+L_{11} \bar{q}^{(1)}(-1) \bar{q}^{(2)}(-1)\right\} \\
g_{21} & =\tau_{0} \bar{D}\left\{2 \left[L _ { 2 0 } \left(W_{20}^{(1)}(-1) \bar{q}^{(1)}(-1)\right.\right.\right. \\
& \left.\left.\left.+2 q^{(1)}(-1) W_{11}^{(1)}(-1)\right)+L_{02} A_{02}\right]+L_{11} a_{11}\right\}
\end{aligned}
$$


where

$$
\begin{aligned}
A_{11}= & q^{(1)}(-1) \bar{q}^{(2)}(-1)+\bar{q}^{(1)}(-1) q^{(2)}(-1), \\
A_{02}= & W_{20}^{(2)}(-1) \bar{q}^{(2)}(-1)+2 q^{(2)}(-1) W_{11}^{(2)}(-1), \\
a_{11}= & W_{20}^{(1)}(-1) \bar{q}^{(2)}(-1)+2 W_{11}^{(1)}(-1) q^{(2)}(-1) \\
& +2 q^{(1)}(-1) W_{11}^{(2)}(-1) \\
& +W_{20}^{(2)}(-1) \bar{q}^{(1)}(-1), \\
q(\theta)= & \left(q^{(1)}(\theta), q^{(2)}(\theta)\right)^{\mathrm{T}}, \\
q^{(1)}(-1)= & e^{-i \tau_{0} \omega_{0}}, \\
q^{(2)}(-1)= & \beta e^{-i \tau_{0} \omega_{0}} .
\end{aligned}
$$

The coefficients $g_{20}, g_{11}$, and $g_{02}$ can be obtained if the parameters in system (4) are given, while the coefficient $g_{21}$ needs to compute $W_{11}(\theta)$ and $W_{20}(\theta)$ by a calculation process similar to [18-20] as follows:

$$
\begin{aligned}
W_{20}(\theta)= & \frac{i g_{20}}{\tau_{0} \omega_{0}} q(0) e^{i \tau_{0} \omega_{0} \theta}+\frac{i \bar{g}_{02}}{3 \tau_{0} \omega_{0}} \bar{q}(0) e^{-i \tau_{0} \omega_{0} \theta} \\
& +E_{1} e^{2 i \tau_{0} \omega_{0} \theta},
\end{aligned}
$$

and

$$
W_{11}(\theta)=\frac{-i g_{11}}{\tau_{0} \omega_{0}} q(0) e^{i \tau_{0} \omega_{0} \theta}+\frac{i \bar{g}_{11}}{\tau_{0} \omega_{0}} \bar{q}(0) e^{-i \tau_{0} \omega_{0} \theta}+E_{2},
$$

where $E_{1}=\left(E_{1}^{(1)}, E_{1}^{(2)}\right)^{\mathrm{T}}, E_{2}=\left(E_{2}^{(1)}, E_{2}^{(2)}\right)^{\mathrm{T}}$ are determined by the following equations, respectively:

$$
\begin{array}{r}
{\left[\begin{array}{cc}
2 i \omega_{0}+k_{2}-m e^{-2 i \tau_{0} \omega_{0}} & n e^{-2 i \tau_{0} \omega_{0}} \\
-k_{3} & 2 i \omega_{0}+k_{4}
\end{array}\right] E_{1}=\left[\begin{array}{c}
h_{1} \\
0
\end{array}\right],} \\
{\left[\begin{array}{cc}
k_{2}-m & n \\
-k_{3} & k_{4}
\end{array}\right] E_{2}=\left[\begin{array}{c}
h_{2} \\
0
\end{array}\right],}
\end{array}
$$

where

$$
\begin{aligned}
h_{1} & =2\left\{L_{20}\left[q^{(1)}(-1)\right]^{2}+L_{02}\left[q^{(2)}(-1)\right]^{2}\right. \\
& \left.+L_{11} q^{(1)}(-1) q^{(2)}(-1)\right\}, \\
h_{2} & =2 L_{20} q^{(1)}(-1) \bar{q}^{(1)}(-1)+2 L_{02} q^{(2)}(-1) \bar{q}^{(2)}(-1) \\
& +L_{11}\left(q^{(1)}(-1) \bar{q}^{(2)}(-1)+\bar{q}^{(1)}(-1) q^{(2)}(-1)\right) .
\end{aligned}
$$

It follows that

$$
\begin{aligned}
& E_{1}^{(1)}=\frac{1}{E}\left|\begin{array}{cc}
h_{1} & n e^{-2 i \tau_{0} \omega_{0}} \\
0 & 2 i \omega_{0}+k_{4}
\end{array}\right|, \\
& E_{1}^{(2)}=\frac{1}{E}\left|\begin{array}{cc}
2 i \omega_{0}+k_{2}-m e^{-2 i \tau_{0} \omega_{0}} & h_{1} \\
-k_{3} & 0
\end{array}\right|,
\end{aligned}
$$

where

$$
E=\left|\begin{array}{cc}
2 i \omega_{0}+k_{2}-m e^{-2 i \tau_{0} \omega_{0}} & n e^{-2 i \tau_{0} \omega_{0}} \\
-k_{3} & 2 i \omega_{0}+k_{4}
\end{array}\right|
$$

Similarly, we can get

$$
\begin{aligned}
& E_{2}^{(1)}=\frac{1}{F}\left|\begin{array}{ll}
h_{2} & n \\
0 & k_{4}
\end{array}\right|, \\
& E_{2}^{(2)}=\frac{1}{F}\left|\begin{array}{cc}
k_{2}-m & h_{2} \\
-k_{3} & 0
\end{array}\right|,
\end{aligned}
$$

where

$$
F=\left|\begin{array}{cc}
k_{2}-m & n \\
-k_{3} & k_{4}
\end{array}\right|
$$

From (56), (57), and (58), we can get $g_{21}$. Furthermore, we can obtain the following values:

$$
\begin{aligned}
c_{1}(0) & =\frac{i}{2 \tau_{0} \omega_{0}}\left(g_{11} g_{20}-2\left|g_{11}\right|^{2}-\frac{\left|g_{02}\right|^{2}}{3}\right)+\frac{g_{21}}{2}, \\
\mu_{2} & =-\frac{\operatorname{Re}\left\{c_{1}(0)\right\}}{\operatorname{Re}\left\{\lambda^{\prime}\left(\tau_{0}\right)\right\}}, \\
\beta_{2} & =2 \operatorname{Re}\left\{c_{1}(0)\right\}, \\
T_{2} & =-\frac{\operatorname{Im}\left\{c_{1}(0)\right\}+\mu_{2} \operatorname{Im}\left\{\lambda^{\prime}\left(\tau_{0}\right)\right\}}{\tau_{0} \omega_{0}} .
\end{aligned}
$$
result.

Through the discussion above, we obtain the following

Theorem 3. For system (4), the coefficient $\mu_{2}$ determines the direction of Hopf bifurcation; it is supercritical (subcritical) if $\mu_{2}>0\left(\mu_{2}<0\right)$; the periodic solutions are stable (unstable) on the manifold if $\beta_{2}<0\left(\beta_{2}>0\right)$; and the period increases (decreases) if $T_{2}>0\left(T_{2}<0\right)$.

\section{Numerical Simulations}

In this section, we present four examples to verify our theoretical results. At same time, the effect of time delay at different situations of negative feedback strength $\Gamma_{2}$ is also investigated.

Example 1. By increasing the negative feedback strength $\Gamma_{2}$, system (4) undergoes a transition from monostability to oscillation and then back to monostability. That is, Hopf bifurcation occurs when the parameter $\Gamma_{2}$ increases to 


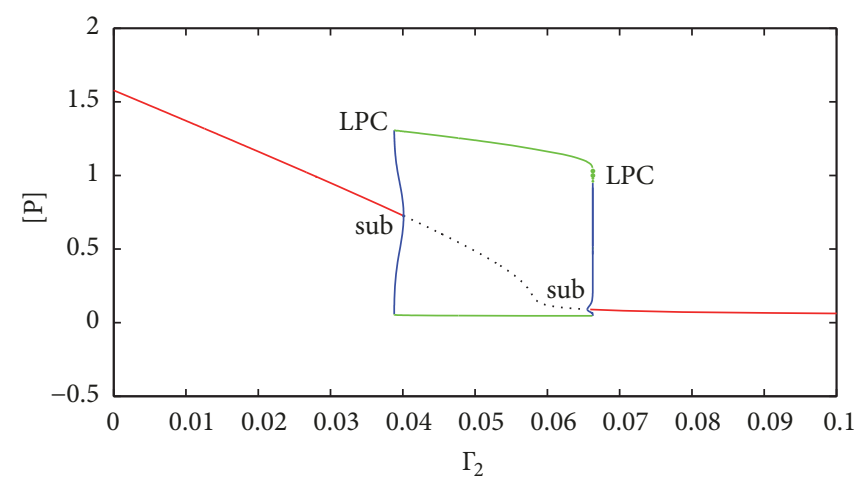

(a)

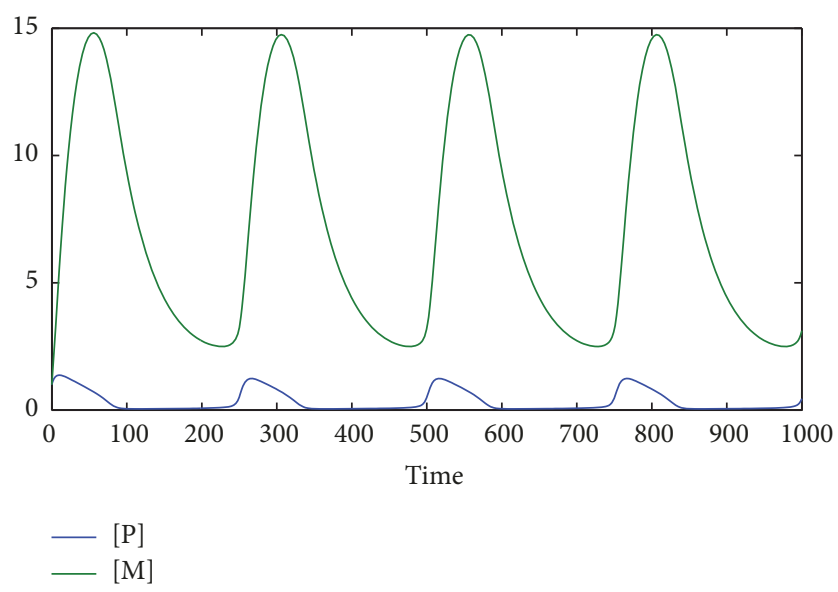

(c)
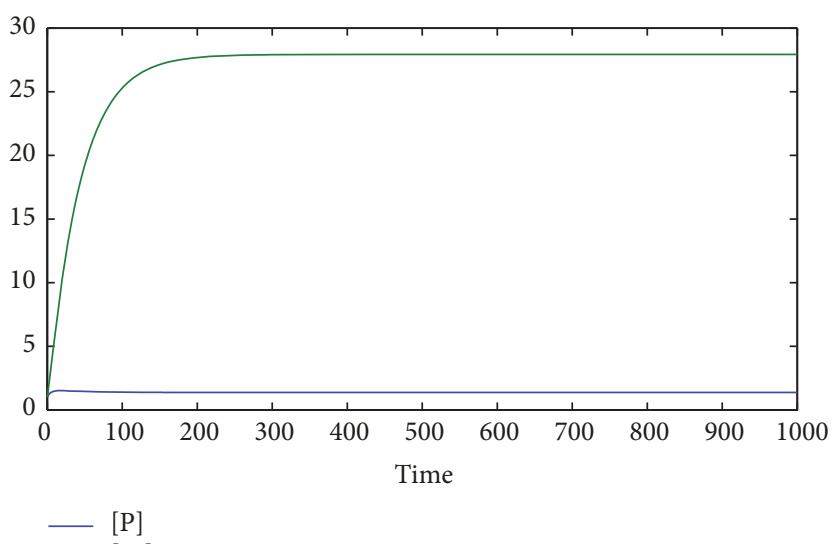

$[\mathrm{M}]$

(b)

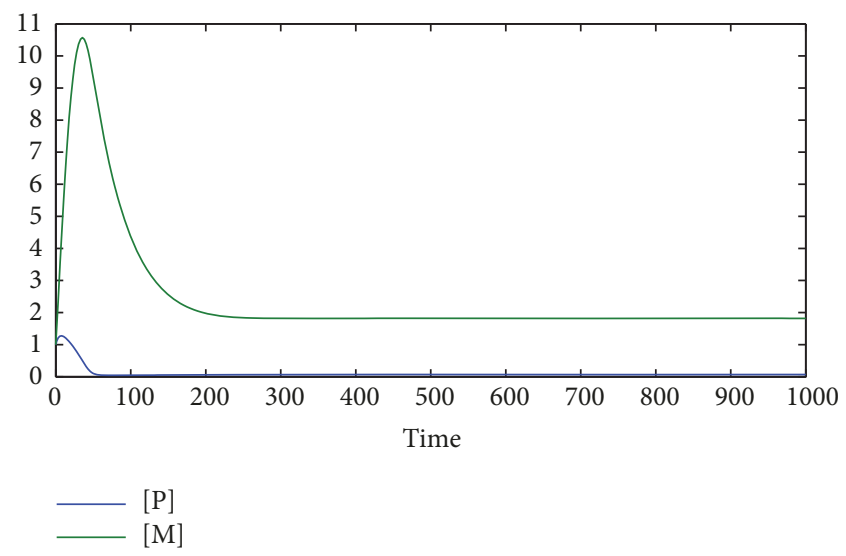

(d)

Figure 2: (a) Bifurcation diagram of [P] with $\Gamma_{2}$ as a bifurcation parameter. The red solid lines depict stable steady states and the black dashed line depicts unstable steady states. The maxima and minima are depicted by the blue dots and green dots for the stable and unstable limit cycle, respectively. The bifurcation points of the subcritical Hopf bifurcation are marked as sub and LPC as fold limit cycle bifurcation points. (b), (c), and (d) are time courses diagram of $P$ and $M$ for the parameter $\Gamma_{2}=0.01(\mathrm{~b}), 0.05(\mathrm{c})$, and 0.09 (d), respectively. The initial conditions: $P(0)=0.1, M(0)=0.1$.

the critical values. Here, we give an example when some important parameters are taken as follows [1,2]:

$$
\begin{aligned}
\alpha_{p} & =0.01, \\
\alpha_{M} & =0.01, \\
k_{p} & =0.4, \\
\Gamma_{1} & =0.1, \\
\beta_{p} & =0.25, \\
k_{M} & =0.4, \\
\beta_{M} & =0.02 .
\end{aligned}
$$

Then, system (4) becomes the following ordinary differential equations:

$$
\begin{aligned}
& \dot{x}=0.01+\frac{0.4 x^{2}}{0.1+x^{2}+\Gamma_{2} y}-0.25 x, \\
& \dot{y}=0.01+0.4 x-0.02 y .
\end{aligned}
$$

Through bifurcation analysis of system (66) in Figure 2(a), we obtain two Hopf bifurcations at $\Gamma_{2}=0.04011$ and 0.06554 when $(\mathrm{H} 2)$ and $(\mathrm{H} 3)$ hold, respectively, as well as limit cycles for $0.04011<\Gamma_{2}<0.06554$ between the two Hopf bifurcations when $\beta_{M}+\beta_{p}-m<0$. Also, the equilibrium $E_{*}$ is asymptotically stable for $\Gamma_{2}<0.04011$ or $\Gamma_{2}>0.06554$. The equilibrium of system (66) at $\Gamma_{2}=0.01$ is stable (see Figure 2(b)) and oscillations of limit cycles at $\Gamma_{2}=0.05$ are presented in Figure 2(c), and then the oscillation behavior vanishes and the equilibrium tends to be stable again at $\Gamma_{2}=0.09($ see Figure $2(\mathrm{~d}))$.

Example 2. We consider dynamic changes induced by time delay $\tau \geq 0$ for the negative feedback strength $\Gamma_{2}=0.01$ when system (4) has a positive equilibrium $E_{1}(1.37151,27.93015)$ at a high steady state in Example 1 in Figure 2. Other parameters are same as Example 1.

In Theorem 2, we get $n_{1}=0.20364, n_{2}=0.00368, \beta_{M}+$ $\beta_{p}-m=k_{2}+k_{4}-k_{1} n_{1}=0.18854>0, \beta_{M}\left(\beta_{p}-m\right)+n k_{M}=$ $k_{2} k_{4}+k_{1} k_{3} n_{2}-k_{1} k_{4} n_{1}=0.00396>0, p=k_{1}^{2} n_{1}^{2}-k_{2}^{2}-k_{4}^{2}=$ 


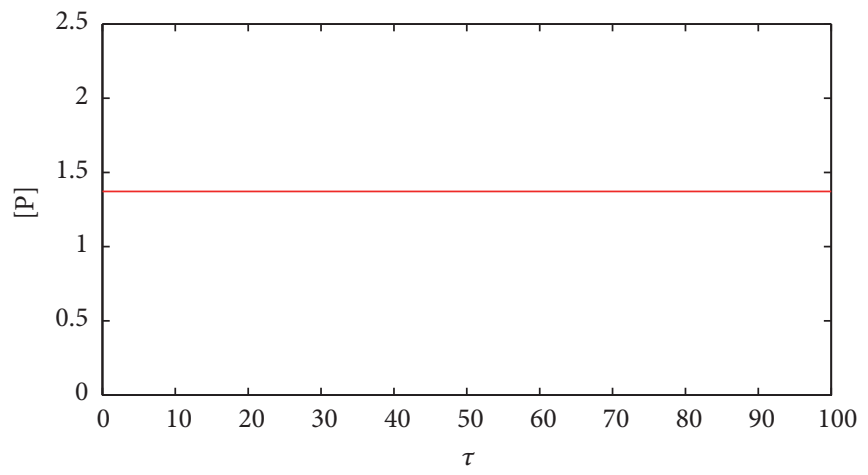

(a)

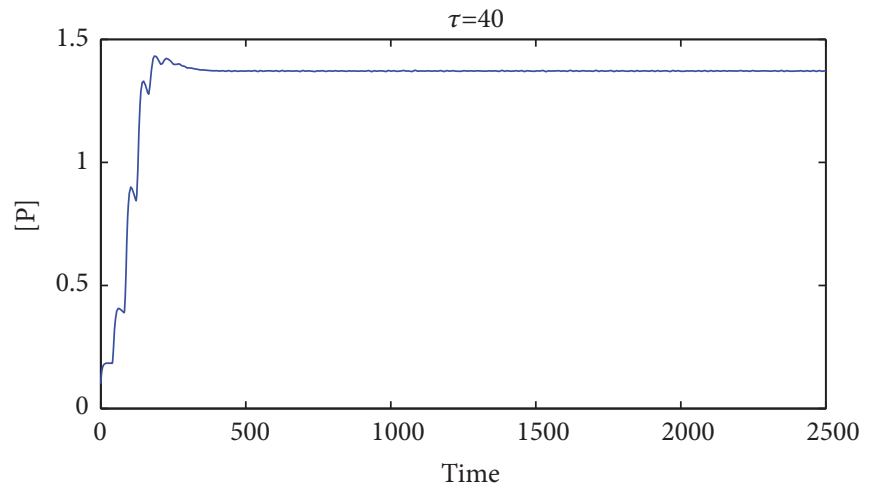

(c)

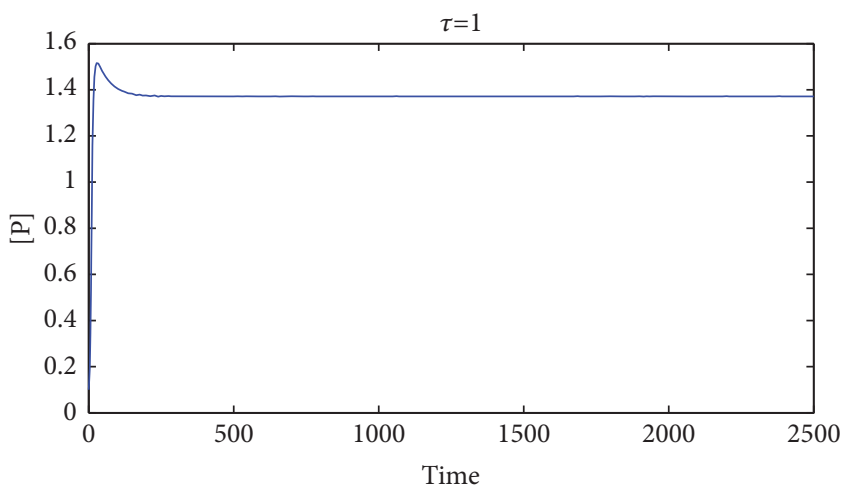

(b)

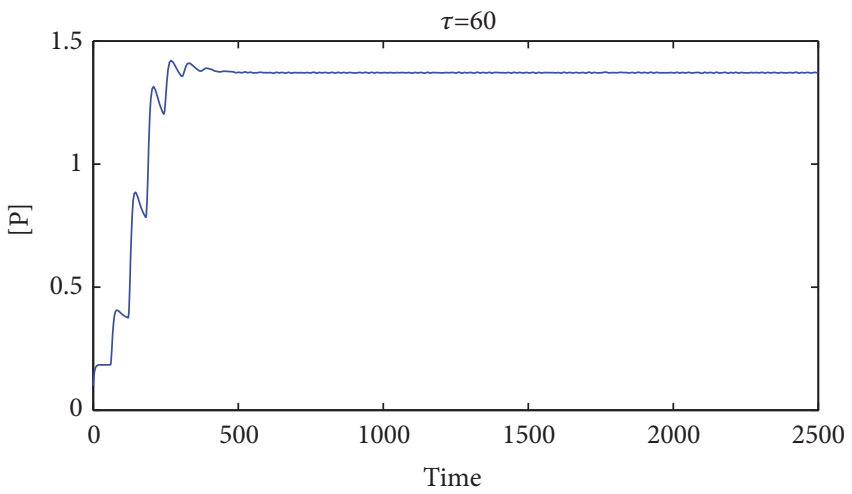

(d)

Figure 3: (a) Bifurcation diagram of [P] with time delay $\tau$ as the bifurcating parameter when fixing the negative feedback strength $\Gamma_{2}=0.01$. (b), (c), and (d) are time courses diagram of $[P]$ for the parameter $\tau=1(\mathrm{~b}), 40(\mathrm{c})$, and 60 (d), respectively. Initial conditions: $P(0)=0.1, M(0)=$ 0.1 .

-0.05626 , and $q=k_{2}^{2} k_{4}^{2}-k_{1}^{2}\left(k_{3} n_{2}-k_{4} n_{1}\right)^{2}=0.00002$. Then conditions (H1), (H2), and (H5) hold. Therefore, we obtain that the steady state of system (4) is stable for all $\tau \geq 0$ (see Figures 3(a), 3(b), 3(c), and 3(d)).

Example 3. A limit cycle and an equilibrium can coexist in system (4) for $\tau=0$ at the negative feedback strength $\Gamma_{2}=$ 0.04 in Figure 2. So system (4) appears to be periodic solution or steady state depending on different initial conditions.

As time delay $\tau$ passes through the critical value $\tau_{0} \approx 2.23$, the periodic solution transits to steady-state solution via Hopf bifurcation when the initial point is chosen near the limit cycle (see Figure 4). Next, we calculate the values of $\beta_{2}$ and $\mu_{2}$ to determine the stability of periodic solutions bifurcating from equilibrium $E_{2}(0.72821,15.06414)$ and direction of the Hopf bifurcation at the critical point $\tau_{0}$. When $\tau=\tau_{0} \approx 2.23$, we can compute $\operatorname{Re}\left(c_{1}(0)\right) \approx-0.20118, \beta_{2} \approx-0.40236$ and $c+d \approx 0.01847>0$ by means of software Maple. Further, we can get $\operatorname{Re}\left(\lambda^{\prime}\left(\tau_{0}\right)\right) \approx 17.96347>0, \mu_{2} \approx 0.0112>0$. By Theorem 3, we know that the direction of Hopf bifurcation is supercritical, and the periodic solutions are stable on the manifold (see Figures 4(a), 4(b), and 4(c)).

Example 4. Fixing the negative feedback strength $\Gamma_{2}=0.07$, system (4) has a positive equilibrium $E_{3}(0.08174,2.13478)$ at a low steady state in Figure 2. The periodic solutions bifurcate from the equilibrium via Hopf bifurcation when time delay $\tau$ increases to the critical value $\tau_{0} \approx 35$ (see Figure 5).

For the arbitrary negative feedback strength $\Gamma_{2} \in(0,0.04)$ and the parameters given in Example 1, system (4) is always stable. For example, at $\Gamma_{2}=0.02$, we have $n_{1}=0.36029$, $n_{2}=0.00728, \beta_{M}+\beta_{p}-m=k_{2}+k_{4}-k_{1} n_{1}=0.12588>0$, $\beta_{M}\left(\beta_{p}-m\right)+n k_{M}=0.00328>0, p=k_{1}^{2} n_{1}^{2}-k_{2}^{2}-k_{4}^{2}=$ -0.04213 , and $q=k_{2}^{2} k_{4}^{2}-k_{1}^{2}\left(k_{3} n_{2}-k_{4} n_{1}\right)^{2}=0.000022$. It means that conditions (H1), (H2), and (H5) hold. By the Theorem 2, we know that system (4) is stable for all $\tau \geq$ 0 . It can explain that delayed negative feedback could not lead to oscillations in the high state in Figure 2. In a word, the time delay leads to oscillation behaviors when system (4) is at the low steady state but not at the high one in Figure 2.

\section{Conclusions and Discussions}

In this paper, the $\mathrm{Myc} / \mathrm{E} 2 \mathrm{~F} / \mathrm{miR}-17-92$ network with time delay is considered. Occurrence of Hopf bifurcations associated with oscillation of the inhibition efficiency parameter $\Gamma_{2}$ and further the time delay $\tau$, respectively, are investigated by 


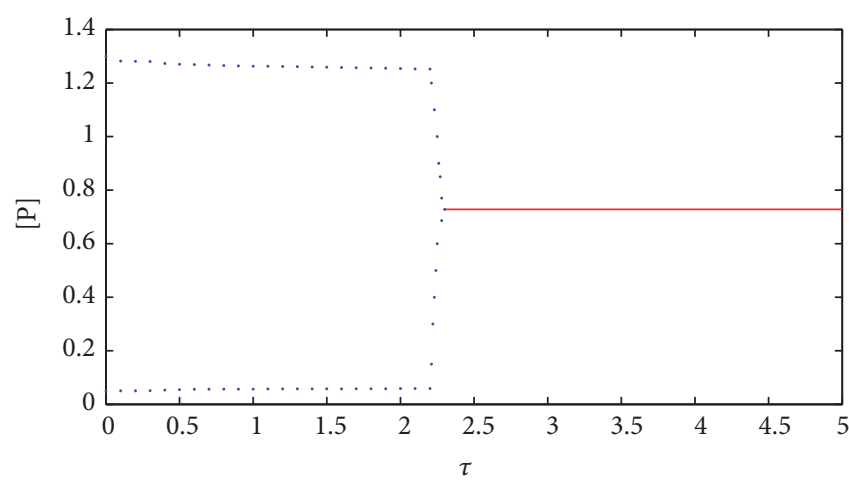

(a)

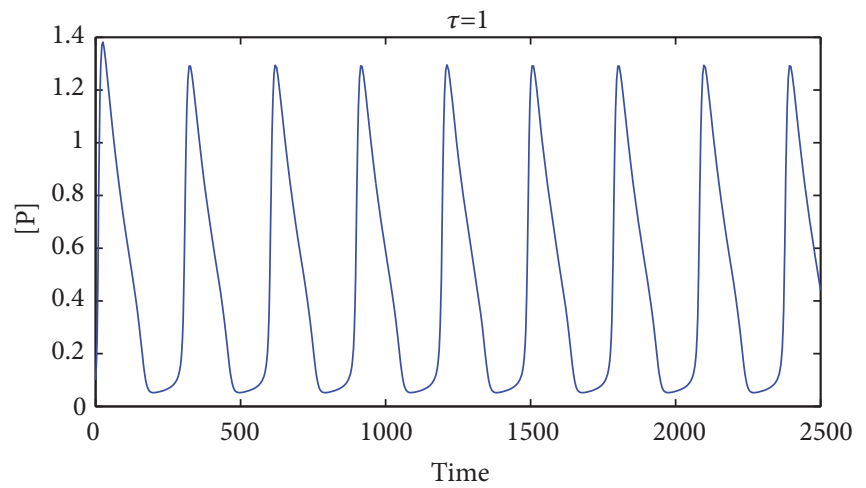

(b)

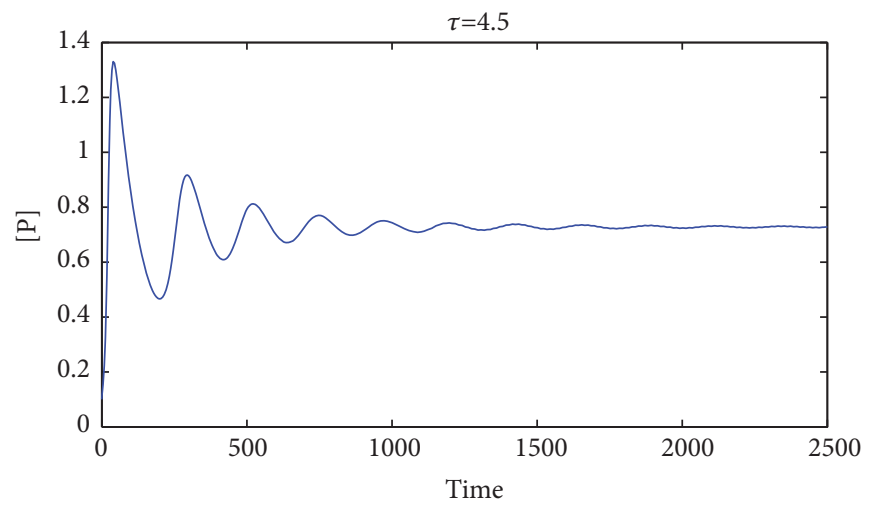

(c)

FIGURE 4: (a) Bifurcation diagram of [P] with time delay $\tau$ as the bifurcating parameter when fixing the negative feedback strength $\Gamma_{2}=$ 0.04. The stable steady state is depicted by the red solid line, and the maxima and minima are depicted by the blue dots for the stable limit cycle. (b) and (c) are time courses diagram of $[P]$ for the parameter $\tau=1$ (b) and 4.5 (c), respectively. Initial conditions: $P(0)=0.1, M(0)=$ 0.1 .

combined local stability and bifurcation theory with numerical simulations. Furthermore, the direction and stability of Hopf bifurcation are also studied by the center manifold theorem and normal form method as well as a numerical example supporting the results.

We find that the time delay has a destabilizing role by choosing appropriate parameters in this network. Besides that, initial condition is important as periodic solution and steady state coexist under some circumstances (see Figure 2). If the initial values are chosen near the positive equilibrium, the steady state of system (4) remains unchanged when the parameter $\tau$ less than the critical value $\tau_{0}$. However, if the initial values are chosen near the limit cycle, system (4) appears to be periodic solution and then transits to steady-state solution via Hopf bifurcation of time delay $\tau$ (see Figure 4). With the negative feedback strength $\Gamma_{2}$ increasing, system (4) undergoes a transition from the high steady state to oscillations and then to low steady state (see Figure 2(a)). We consider the effect of time delay on the dynamics of the network at every different state. When the negative feedback strength $\Gamma_{2}$ is chosen at the high steady states, there will be no periodic oscillations with increasing time delay $\tau$ (see Figure 3 ). However, when the negative feedback strength $\Gamma_{2}$ is chosen between the two Hopf bifurcation points in Figure 2(a), the periodic solutions transit to steady-state solutions via Hopf bifurcation with increasing time delay (see Figure 4). The periodic solutions bifurcate from the equilibrium of system (4) with increasing time delay $\tau$ when the negative feedback strength $\Gamma_{2}$ is chosen at the low steady states (see Figure 5). The values of the parameters are chosen in the special way above or they need to satisfy the conditions in Theorem 2. If (H1), (H2), and (H5) hold, then the equilibrium of system (4) is asymptotically stable for all $\tau \geq 0$; if (H1), (H2), and (H4) hold, the equilibrium is locally asymptotically stable for $0 \leq \tau<\tau_{0}$; moreover, if $c+d \neq 0$, system (4) undergoes a Hopf bifurcation at the equilibrium when $\tau=\tau_{0}$. At the same time, the direction and stability of Hopf bifurcation can be determined by Theorem 3. This work can further provide a theoretic instruction for exploring the dynamics of the network.

Noise is inevitable in gene regulatory networks and plays important roles in circuits' dynamics. It may induce bistability, oscillations, and bifurcations which are not present in the deterministic model [7, 21, 22]. How does stochastic noise affect dynamics of the network with time delay? This is a very valuable and worth exploring problem. We leave it as the future work. 


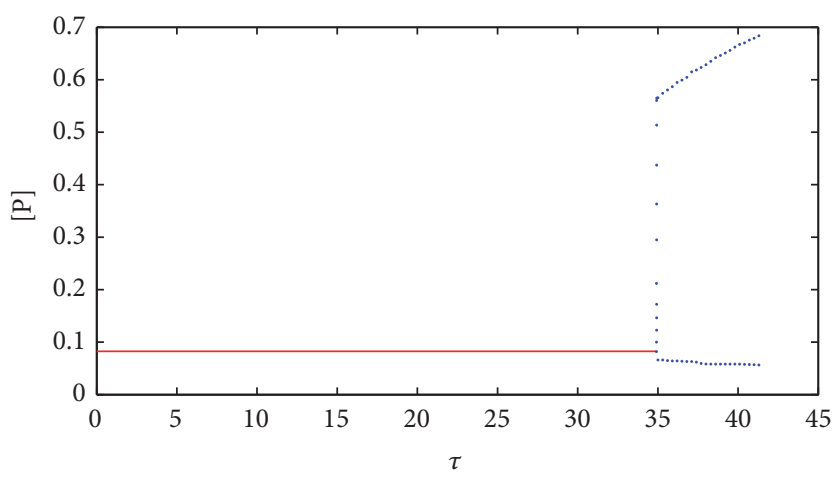

(a)

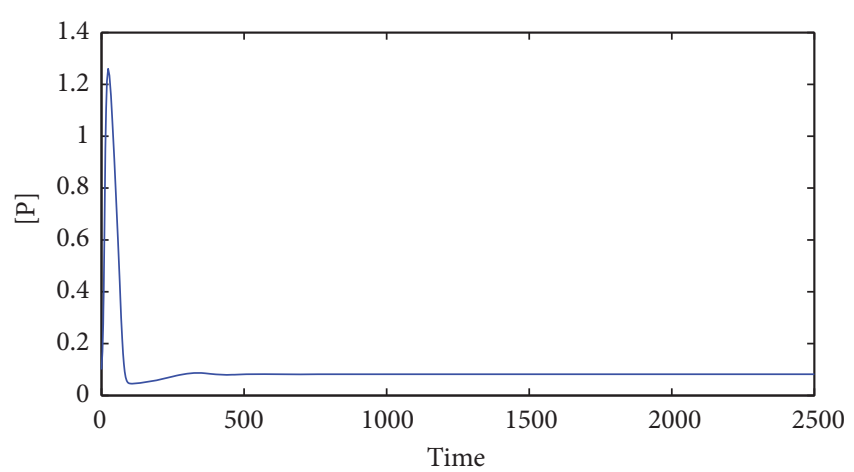

(b)

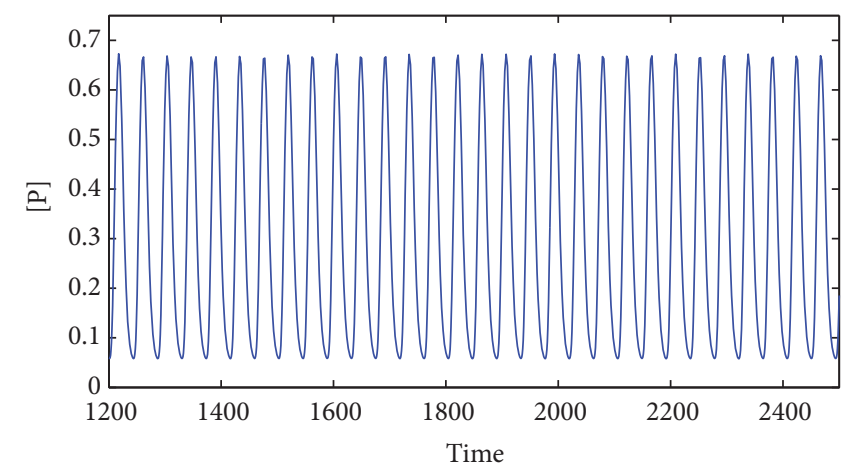

(c)

FiguRE 5: (a) Bifurcation diagram of [P] with time delay $\tau$ as the bifurcating parameter when fixing the negative feedback strength $\Gamma_{2}=0.07$. The red solid line depicts stable steady states and the blue dots depict the maxima and minima for the stable limit cycle. (b) and (c) are time courses diagram of $[P]$ for the parameter $\tau=1(\mathrm{~b})$ and 40 (c), respectively. Initial conditions: $P(0)=0.1, M(0)=0.1$.

\section{Data Availability}

The data used to support the findings of this study are available from the corresponding author upon request.

\section{Conflicts of Interest}

The authors declare that they have no conflicts of interest.

\section{Acknowledgments}

This work is supported by the National Natural Science Foundation of China (nos. 11872084, 11472009, and 11801224).

\section{References}

[1] B. D. Aguda, Y. Kim, M. G. Piper-Hunter, A. Friedman, and C. B. Marsh, "MicroRNA regulation of a cancer network: consequences of the feedback loops involving miR-17-92, E2F, and Myc," Proceedings of the National Acadamy of Sciences of the United States of America, vol. 105, no. 50, pp. 19678-19683, 2008.

[2] Y. Li, Y. Li, H. Zhang, Y. Chen, and R. J. Morris, "MicroRNAMediated Positive Feedback Loop and Optimized Bistable Switch in a Cancer Network Involving miR-17-92," PLoS ONE, vol. 6, no. 10, p. e26302, 2011.
[3] H. Zhang, Y. Chen, Y. Chen, and K. Selvarajoo, "Noise Propagation in Gene Regulation Networks Involving Interlinked Positive and Negative Feedback Loops," PLoS ONE, vol. 7, no. 12, p. e51840, 2012.

[4] D. Liu, X. Chang, Z. Liu, L. Chen, R. Wang, and E. Ben-Jacob, "Bistability and Oscillations in Gene Regulation Mediated by Small Noncoding RNAs," PLoS ONE, vol. 6, no. 3, p. e17029, 2011.

[5] A. Zinovyev, N. Morozova, A. N. Gorban, and A. Harel-Belan, "Mathematical modeling of microRNA-mediated mechanisms of translation repression," Advances in Experimental Medicine and Biology, vol. 774, pp. 189-224, 2013.

[6] M. Lu, M. K. Jolly, H. Levine, J. N. Onuchic, and E. Ben-Jacob, "MicroRNA-based regulation of epithelial-hybridmesenchymal fate determination," Proceedings of the National Acadamy of Sciences of the United States of America, vol. 110, no. 45, pp. 18144-18149, 2013.

[7] P. Zhou, S. Cai, Z. Liu, and R. Wang, "Mechanisms generating bistability and oscillations in microRNA-mediated motifs," Physical Review E: Statistical, Nonlinear, and Soft Matter Physics, vol. 85, no. 4, p. 041916, 2012.

[8] A. Wan and X. Zou, "Hopf bifurcation analysis for a model of genetic regulatory system with delay," Journal of Mathematical Analysis and Applications, vol. 356, no. 2, pp. 464-476, 2009.

[9] J. Yu and M. Peng, "Local Hopf bifurcation analysis and global existence of periodic solutions in a gene expression model with delays," Nonlinear Dynamics, vol. 86, no. 1, pp. 1-12, 2016. 
[10] M. Bodnar and A. Bartłomiejczyk, "Stability of delay induced oscillations in gene expression of Hesl protein model," Nonlinear Analysis: Real World Applications, vol. 13, no. 5, pp. 22272239, 2012.

[11] X. P. Wu and M. Eshete, "Bifurcation analysis for a model of gene expression with delays," Communications in Nonlinear Science and Numerical Simulation, vol. 16, no. 2, pp. 1073-1088, 2011.

[12] Z.-R. Xie, H.-T. Yang, W.-C. Liu, and M.-J. Hwang, “The role of microRNA in the delayed negative feedback regulation of gene expression," Biochemical and Biophysical Research Communications, vol. 358, no. 3, pp. 722-726, 2007.

[13] X.-J. Tian, X.-P. Zhang, F. Liu, and W. Wang, "Interlinking positive and negative feedback loops creates a tunable motif in gene regulatory networks," Physical Review E: Statistical, Nonlinear, and Soft Matter Physics, vol. 80, no. 1, p. 011926, 2009.

[14] B. Huang, X. Tian, F. Liu, and W. Wang, "Impact of time delays on oscillatory dynamics of interlinked positive and negative feedback loops," Physical Review E: Statistical, Nonlinear, and Soft Matter Physics, vol. 94, no. 5, p. 052413, 2016.

[15] Y. Suzuki, M. Lu, E. Ben-Jacob, and J. N. Onuchic, "Periodic, Quasi-periodic and Chaotic Dynamics in Simple Gene Elements with Time Delays," Scientific Reports, vol. 6, p. 21037, 2016.

[16] B. Novák and J. J. Tyson, "Design principles of biochemical oscillators," Nature Reviews Molecular Cell Biology, vol. 9, no. 12, pp. 981-991, 2008.

[17] B. D. Hassard, N. D. Kazarinoff, and Y.-H. Wan, Theory and Applications of Hopf Bifurcation, Cambridge University Press, Cambridge, UK, 1981.

[18] W. Yu and J. Cao, "Hopf bifurcation and stability of periodic solutions for van der Pol equation with time delay," Nonlinear Analysis: Theory, Methods \& Applications, vol. 62, no. 1, pp. 141165, 2005.

[19] W. W. Yu and J. D. Cao, "Stability and Hopf bifurcation analysis on a four-neuron BAM neural network with time delays," Physics Letters A, vol. 351, no. 1, pp. 64-78, 2006.

[20] Y. L. Song and S. L. Yuan, "Bifurcation analysis in a predatorprey system with time delay," Nonlinear Analysis: Real World Applications, vol. 7, no. 2, pp. 265-284, 2006.

[21] A. Lipshtat, A. Loinger, N. Q. Balaban, and O. Biham, "Genetic toggle switch without cooperative binding," Physical Review Letters, vol. 96, no. 18, p. 188101, 2006.

[22] T. B. Kepler and T. C. Elston, "Stochasticity in transcriptional regulation: Origins, consequences, and mathematical representations," Biophysical Journal, vol. 81, no. 6, pp. 3116-3136, 2001. 


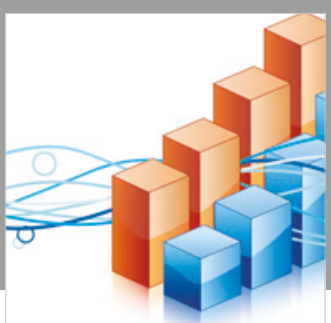

Advances in

Operations Research

\section{-n-m}
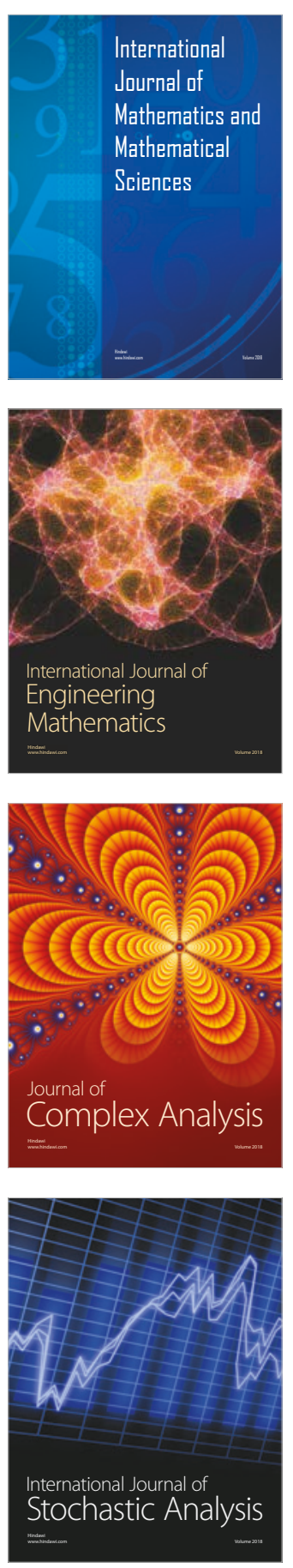
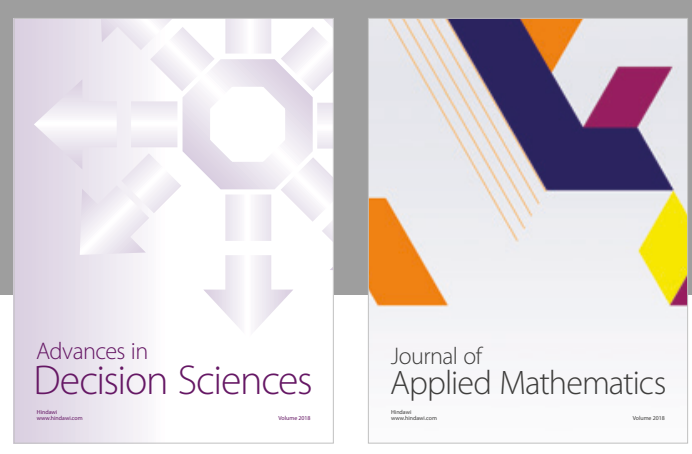

Journal of

Applied Mathematics
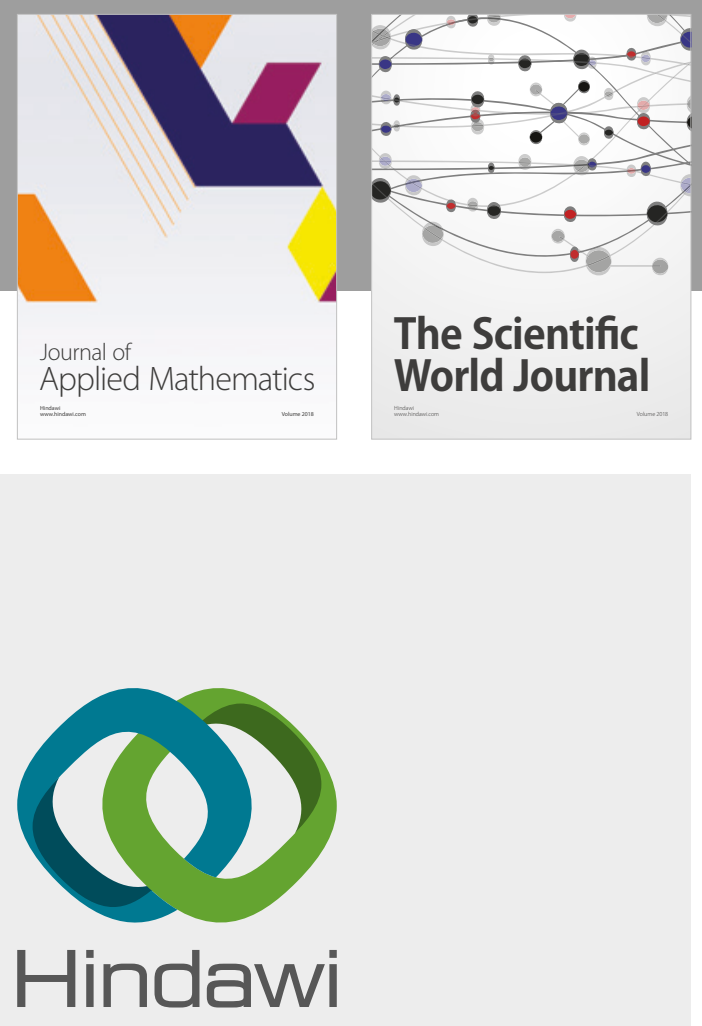

Submit your manuscripts at

www.hindawi.com

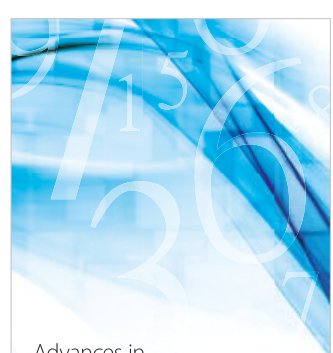

Advances in
Numerical Analysis
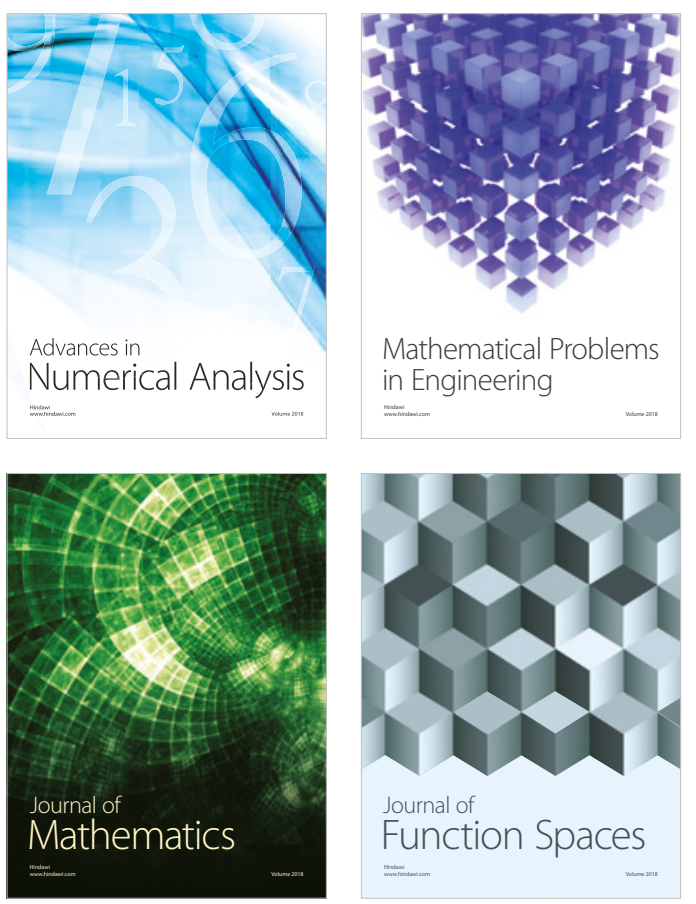

Mathematical Problems in Engineering

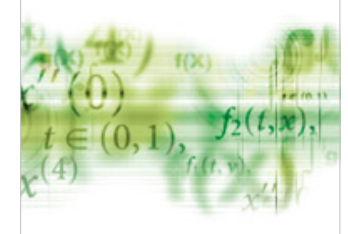

International Journal of

Differential Equations

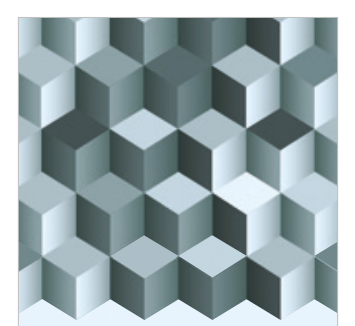

Journal of

Function Spaces

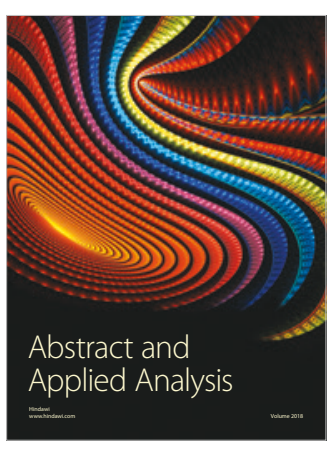

The Scientific

World Journal

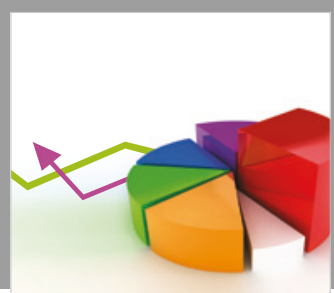

Journal of

Probability and Statistics
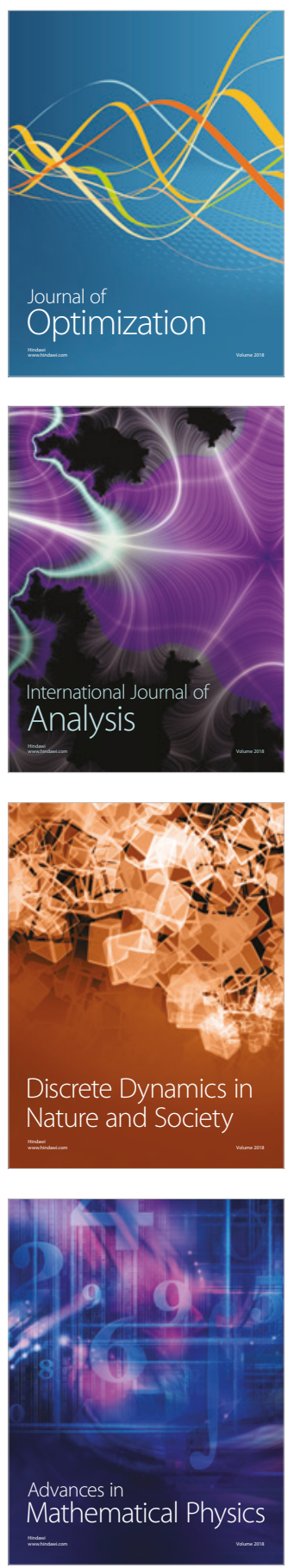